\title{
Analytical model for modulation of diffuse light by pulsed ultrasonic waves
}

Sava Sakadzic, Lihong V. Wang

Sava Sakadzic, Lihong V. Wang, "Analytical model for modulation of diffuse light by pulsed ultrasonic waves," Proc. SPIE 5697, Photons Plus Ultrasound: Imaging and Sensing 2005: The Sixth Conference on Biomedical Thermoacoustics, Optoacoustics, and Acousto-optics, (25 April 2005); doi: $10.1117 / 12.589432$

SPIE. Event: SPIE BiOS, 2005, San Jose, CA, United States 


\title{
Analytical model for modulation of diffuse light by pulsed ultrasonic waves
}

\author{
Sava Sakadžić and Lihong V. Wang \\ Optical Imaging Laboratory, Department of Biomedical Engineering, Texas A\&M University, \\ College Station, Texas 77843-3120
}

\begin{abstract}
We present an analytical solution for the acousto-optical modulation of multiply scattered light in a medium irradiated with a train of ultrasound pulses. Previous theory is extended to cases where the ultrasound induced optical phase increments between the different scattering events are strongly correlated. The relation between the ultrasound induced motions of the fluid and the optical scatterers is generalized, and it is shown that correlation exists between the optical phase increments that are due to the scatterer movement and to the modulation of the optical index of refraction. Finally, it is shown that compared with the spectrum of the ultrasound pulses, the power spectral density of the acousto-optically modulated light is strongly attenuated toward the higher ultrasound frequencies.
\end{abstract}

Keywords: ultrasonic modulation, acousto-optical tomography, multiply scattered light, autocorrelation function, turbid medium

\section{INTRODUCTION}

The optical properties of soft biological tissues in the visible and near-infrared regions are related to the molecular tissue structure. Radiation at these wavelengths is nonionizing, and it has significant potential for the functional imaging and detection of tissue abnormalities. Much effort has been made to develop new soft tissue imaging modalities based on visible and near-infrared radiation.

Ultrasound-modulated optical tomography is a hybrid technique, proposed to provide better resolution for the optical imaging of soft biological tissue by combining ultrasonic resolution and optical contrast. In this technique, ${ }^{1,2}$ optical radiation, which has high temporal coherence, and ultrasound are applied simultaneously to soft biological tissue. The intensity of the ultrasound-modulated optical radiation is measured to provide information about the optical properties of the tissue spatially localized in the region of the interaction of the ultrasonic and electromagnetic waves.

In spite of a variety of different experimental configurations that have been invented to efficiently measure the ultrasonically modulated component of the optical intensity emerging from the biological tissue, the exact nature of the acousto-optical effect in a highly optically scattering medium is still not totally understood due to the complicated light-ultrasound interaction in the presence of optical scatterers. Simple theories in the optical diffusion regime under the week scattering approximation have been developed ${ }^{3-7}$ that include one or both of the main mechanisms of modulation. Mechanism 1 is optical phase variations that are due to the ultrasonically induced movement of the optical scatterers,,${ }^{3,4}$ and mechanism 2 is optical phase variations that are due to ultrasonically induced changes in the optical index of refraction. Mechanism 2 was first modelled by Wang ${ }^{5}$ combined with mechanism 1. Subsequently, the model was extended to account for anisotropic optical scattering, Brownian motion, and optical absorption. ${ }^{7}$ Due to the limited number of physical configurations where the probability density function of the optical pathlength is analytically known, only slab transmission ${ }^{5}$ and reflection ${ }^{8}$ geometries are being analytically studied at present. However, the model can be easily incorporated into a Monte Carlo algorithm, ${ }^{6,7}$ offering the possibility of exploring a wide spectrum of geometries.

The existing theoretical model was developed for the interaction of a plane, monochromatic (CW) ultrasound wave with diffused light in an infinite scattering medium, neglecting the polarization effects. It is assumed that the ratio of the optical transport mean free path $l_{t r}$ to the ultrasonic wavelength $\lambda_{a}$ is large enough that the ultrasound induced optical phase increments associated with different scattering events are weakly correlated. ${ }^{4}$ 
However, this assumption may not be valid in cases where broadband pulsed ultrasound is applied, which is a promising direction in development of soft tissue imaging technology based on an acousto-optical effect. ${ }^{9-11}$

In this work, we extended present theory to cases where broadband ultrasound pulses are interacting with diffused light. In Sec. 2.1, we generalize the relation between the ultrasound induced optical scatterer movement and the fluid displacement in accordance with the analytical solution for a small rigid sphere oscillation in a viscous fluid. In Sec. 2.2, we develop an expression for the time averaged temporal autocorrelation function of the electrical field component associated with the optical paths of length $s$ in turbid media, when an infinite train of ultrasonic pulses traverse the media. The approximate similarity relation is valid for a broad range of $l_{t r} / \lambda_{a}$ ratios. We show that, in general, a correlation exists between the phase increments due to scatterer displacement and phase increments due to index of refraction changes even when the value of $l_{t r} / \lambda_{a}$ is large. In Sec. 3, we explore the influence of ultrasound frequencies on the behavior of acousto-optically modulated optical intensity. In Sec. 4, we present a complete solution for acousto-optical modulation for a few distinct profiles of ultrasound pulses in slab transmission geometry. Finally, a summary of the results is presented.

\section{TEMPORAL AUTOCORRELATION FUNCTION OF THE ELECTRICAL FIELD}

\subsection{Ultrasound induced movement of the optical scatterers}

In general, the equations governing the ultrasound induced motion of the particle in a fluid are complex. Based on the analytical model for oscillations of a small rigid spherical particle in a viscous flow, with no-slip conditions applied on the surface of the particle ${ }^{12-15}$ the relation between the Fourier transform of fluid velocity $\tilde{u}(f)$ and the Fourier transform of particle velocity $\tilde{v}(f)$ is given by $\tilde{v}(f)=\tilde{u}(f) Y\left(f_{r}, \gamma\right)$, where

$$
Y\left(f_{r}, \gamma\right)=\frac{1-i f_{r}-(i-1)\left(3 f_{r} / 2\right)^{1 / 2}}{1-i(2 \gamma+1) f_{r} / 3-(i-1)\left(3 f_{r} / 2\right)^{1 / 2}} .
$$

In Eq. 1 the relative ultrasonic frequency, $f_{r}=f / \nu_{0}$, is calculated in respect to $\nu_{0}=3 \eta_{k} /\left(2 \pi a_{0}^{2}\right) ; i=\sqrt{-1}$ is imaginary unit; $a_{0}$ is sphere radius; $\eta_{k}$ is kinematic viscosity; and $\gamma=\hat{\rho} / \rho$ is the relative sphere density where $\hat{\rho}$ and $\rho$ are densities of the sphere and the fluid, respectively.

When the relative density of the particle is low $(\gamma<1)$, the amplitude of the particle oscillation is greater than the amplitude of the fluid oscillation, and the phase of the particle oscillation precedes the phase of the fluid oscillation. However, it is expected that in soft biological tissue, optical scatterers are just slightly heavier than surrounding fluid, and they should follow closely the fluid movement.

\subsection{Temporal autocorrelation function for the train of ultrasound pulses}

In this model, we consider the independent multiple scattering of temporarily coherent diffused light in a homogeneous scattering medium in a case of isotropic optical scattering. We neglect the polarization effects and assume that the optical wavelength $\lambda_{0}$ is much smaller than the scattering mean free path $l$. We also assume that an ultrasonic wave is propagating unperturbed along the $x$ axis without attenuation. The acoustical pressure in the medium is given by $P(\vec{r}, t)=P_{0} f(x, t)$, where $P_{0}$ is the pressure amplitude, and the pressure time dependence is represented by the function $f(x, t)$. Analogous to previous work ${ }^{16,17}$ where the acousto-optical effect caused by pulsed ultrasound is analyzed in a clear medium, we assume that the pressure time dependence function $f(x, t)$ represents an infinite train of ultrasound pulses

$$
f(x, t)=\sum_{n=-\infty}^{+\infty} f_{0}\left(x-v_{a} t-n v_{a} T\right),
$$

where $v_{a}$ is the ultrasonic speed, and $T$ is the time period between ultrasound pulses.

We assume in this simple model that due to the weak scattering approximation $\left(l / \lambda_{0} \gg 1\right)$ the fields belonging to different random paths add incoherently in the average, and only the photons travelling along the same path of length $s$ contribute to the autocorrelation function..$^{3,18-21}$ Consequently, the time averaged autocorrelation function of the electrical field can be written as 


$$
\Gamma(\tau)=\int_{0}^{\infty} p(s) \Gamma_{s}(\tau) d s
$$

where $p(s)$ is the probability density function that the optical paths have length $s$, and $\Gamma_{s}(\tau)$ is the time averaged autocorrelation function of the electrical field associated with the paths of length $s$.

To obtain the value of $\Gamma_{s}(\tau)$ we first consider the phase $\varphi_{s}$ of the electrical field component accumulated along the optical paths of length $s$ in optically diffusive media. The value of the electrical field component in analytic signal representation is then proportional to $\exp \left[-i\left(\omega_{0} t-\varphi_{s}\right)\right]$, where $\omega_{0}=2 \pi f_{0}$, and $f_{0}$ is the optical frequency of the incident monochromatic light.

For an optical path of length $s$, which begins at $\vec{r}_{0}$ and ends at $\vec{r}_{N+1}$ and has $N$ scatterers at positions $\vec{r}_{1}, \ldots, \vec{r}_{N}$, the value of the accumulated optical phase is approximately equal to

$$
\varphi_{s}(H, t)=k_{0} n_{0} \sum_{i=1}^{N}\left(\chi_{i}-\chi_{i+1}\right) \varepsilon_{i}(t)+\frac{1}{2} k_{0} n_{0} M \sum_{i=0}^{N} \int_{\vec{r}_{i}}^{\vec{r}_{i+1}} f(\vec{r}, t) d r
$$

In Eq. 4, integrations in the last term are performed along the straight lines which connect consecutive scatterers; $k_{0}=2 \pi / \lambda_{0}$ is the magnitude of the optical wave vector, $\chi_{i+1}=\cos \left(\theta_{i+1}\right)$ where $\theta_{i+1}$ is the angle between ultrasound wave-vector $\vec{k}_{a}$ and the vector $\vec{l}_{i+1}=\vec{r}_{i+1}-\vec{r}_{i}$ which connects two consecutive scatterers; and $\varepsilon_{j}(t)$ is the projection of the ultrasound induced displacement of the $j$ th particle $\vec{\varepsilon}_{j}(t)$ at time $t$ in the ultrasound propagation direction. We also assume that the perturbation of the optical index of refraction $n(x, t)$ due to the ultrasound is small, so that we can write $n(x, t) \approx n_{0}[1+M f(x, t) / 2]$, where modulation coefficient $M$ is equal to $2 \eta P_{0} / \rho v_{a}^{2}$, and $\eta=\rho \partial n / \partial \rho$ is the elasto-optic coefficient (we assume for water $\eta \approx 0.32$ ). The term $H$ represents the set of random variables $\left\{\overrightarrow{r_{0}}, \chi_{1}, l_{1}, \ldots, \chi_{N+1}, l_{N+1}\right\}$ associated with the paths of length $s$ with $N$ scatterers. As in the previous work, ${ }^{7}$ we assume that the correlation between the lengths of free paths $l_{j}$ is weak, since the number of steps $\mathrm{N}$ in each photon path in the diffusion regime is much larger than unity. The probability density functions (PDF) of the first scatterer position and the cosines of the starting angles $\chi_{i}$ are uniform. Also the PDF of the optical path length between two scattering events is given by $p\left(l_{j}\right)=l^{-1} \exp \left[-l_{j} / l\right]$, where $l$ is the mean optical free path, and the PDF of having the $N$ scattering events along a path of length $s$ is given by $p_{N}(s)=(s / l)^{N} \exp [-s / l] / N !$.

Now, we calculate the power spectral density of the optical intensity as a Fourier transform of the time averaged autocorrelation function. ${ }^{22}$ We adopt the notation $\Delta \varphi_{s}=\varphi_{s}(H, t+\tau)-\varphi_{s}(H, t)$, such that the time averaged autocorrelation function $\Gamma_{s}(\tau)$ is expressed as $\Gamma_{s}(\tau)=\exp \left[-i \omega_{0} \tau\right]\left\langle\exp \left[i \Delta \varphi_{s}\right]\right\rangle_{t, H, N}$, where \langle\rangle$_{t, H, N}$ represents averaging over time, all the random variables in $H$, and finally averaging over the realizations of the number of scattering events $N$ along the paths of length $s$.

We proceed by representing the $\Delta \varphi_{s}$ with the help of the Eq. 4 as $\Delta \varphi_{s}=\Delta \varphi_{s, n}+\Delta \varphi_{s, d}$, where $\Delta \varphi_{s, n}$ is associated with index of refraction changes along the optical path

$$
\Delta \varphi_{s, n}=\frac{1}{2} k_{0} n_{0} M \sum_{i=0}^{N} \int_{\vec{r}_{i}}^{\vec{r}_{i+1}} \Delta f(\vec{r}, t, \tau) d r
$$

where $\Delta f(\vec{r}, t, \tau)=f(\vec{r}, t+\tau)-f(\vec{r}, t)$. Similarly, term $\Delta \varphi_{s, d}$ is associated with the ultrasound induced movement of the scatterers

$$
\Delta \varphi_{s, d}=k_{0} n_{0} \sum_{j=1}^{N}\left(\chi_{j}-\chi_{j+1}\right) \Delta \varepsilon_{j}(t, \tau)
$$


where $\Delta \varepsilon_{j}(t, \tau)=\varepsilon_{j}(t+\tau)-\varepsilon_{j}(t)$.

The function $f(x, t)$ represents the acoustical pressure time dependence [Eq. 2]. Its representation using the Fourier spectral components is given by

$$
f(x, t)=\frac{1}{v_{a} T} \sum_{n=-\infty}^{+\infty} \tilde{f}_{0}\left(\frac{n}{v_{a} T}\right) \exp \left[-i n\left(k_{a} x-\omega_{a} t\right)\right]
$$

where $k_{a}=2 \pi /\left(v_{a} T\right)$ and $\omega_{a}=2 \pi / T$ are respectively the ultrasonic wave vector magnitude and angular frequency associated with the period between ultrasonic pulses $T$. In Eq. 7, the Fourier transform $\tilde{f}_{0}(\nu)$ of the ultrasonic pulse shape function $f_{0}\left(x-v_{a} t\right)$ is $\tilde{f}_{0}(\nu)=\int_{-\infty}^{+\infty} f_{0}(u) \exp [i 2 \pi \nu u] d u$.

To obtain the expression for the displacement of the scatterers, we assume that at each ultrasonic frequency $f$ in a spectrum of the infinite train of ultrasonic pulses, the relation given by Eq. 1 is satisfied. For simplicity, we represent the variable $Y\left(f_{r}, \gamma\right)$ as a product $Y\left(f_{r}, \gamma\right)=S\left(f_{r}\right) \exp \left[i \phi\left(f_{r}\right)\right]$, where $S\left(f_{r}\right)$ is the amplitude and $\phi\left(f_{r}\right)$ is the phase of the scatterer velocity deviation from the fluid velocity. Then, the relation between the Fourier transforms of the scatterer velocity and the fluid velocity becomes $\tilde{v}(f)=\tilde{u}(f) S\left(f_{r}\right) \exp \left[i \phi\left(f_{r}\right)\right]$. In the further derivations, we will denote with $S_{n}$ and $\phi_{n}$ the values of $S\left(f_{r}\right)$ and $\phi\left(f_{r}\right)$ at ultrasound frequencies equal to $f_{n}=n / T$.

If we assume that the velocity of the fluid is given by $P(x, t) /\left(\rho v_{a}\right)$, the displacement of the $j$ th scatterer can be expressed as

$$
\varepsilon_{j}(t)=-\frac{i P_{0}}{2 \pi \rho v_{a}^{2}} \sum_{\substack{n=-\infty \\ n \neq 0}}^{+\infty} \tilde{f}_{0}\left(\frac{n}{v_{a} T}\right) \frac{S_{n} \exp \left[-i \phi_{n}\right]}{n} \exp \left[-i n\left(k_{a} x_{j}-\omega_{a} t\right)\right]
$$

In Eq. 8 we assumed that no streaming is present in the fluid, so the spectral component associated with $n=0$ (DC component) is excluded from the spectrum. Since the DC component is not playing any role in mechanism 2, it is also excluded from the solution for the phase term $\Delta \varphi_{s, n}$.

By combining the Equations 5, 6, 7, and 8, we obtain expressions for the values of the phase terms $\Delta \varphi_{s, n}$ and $\Delta \varphi_{s, d}$ for the train of ultrasound pulses

$$
\begin{aligned}
\Delta \varphi_{s, n} & =i \frac{\Lambda}{4 \pi} \sum_{\substack{n=-\infty \\
n \neq 0}}^{+\infty} \frac{\eta}{n} \tilde{f}_{0}\left(\frac{n}{v_{a} T}\right) \exp \left[i n \omega_{a} t\right]\left\{\exp \left[i n \omega_{a} \tau\right]-1\right\} \sum_{j=0}^{N} \frac{\exp \left[-i n k_{a} x_{j+1}\right]-\exp \left[-i n k_{a} x_{j}\right]}{\chi_{j+1}} \\
\Delta \varphi_{s, d} & =i \frac{\Lambda}{4 \pi} \sum_{\substack{n=-\infty \\
n \neq 0}}^{+\infty} \frac{-S_{n} e^{-i \phi_{n}}}{n} \tilde{f}_{0}\left(\frac{n}{v_{a} T}\right) \exp \left[i n \omega_{a} t\right]\left\{\exp \left[i n \omega_{a} \tau\right]-1\right\} \sum_{j=1}^{N}\left(\chi_{j}-\chi_{j+1}\right) \exp \left[-i n k_{a} x_{j}\right]
\end{aligned}
$$

where $\Lambda=2 n_{0} k_{0} P_{0} /\left(\rho v_{a}^{2}\right)$.

We assume, like in the previous work, ${ }^{5}$ that the total phase perturbation $\Delta \varphi_{s}$ due to the ultrasound is much less than one radian. In that case, it is sufficient to consider only the first two terms in the development of the exponential function in the expression of the autocorrelation function $\left\langle\exp \left[i \Delta \varphi_{s}\right]\right\rangle_{t, H, N} \approx 1-\left\langle\Delta \varphi_{s}^{2}\right\rangle_{t, H, N} / 2$.

To obtain the expression for $\left\langle\Delta \varphi_{s}^{2}\right\rangle_{t, H, N}$, we first split the whole term into three parts associated with the ultrasound induced optical index of refraction changes, with the displacements of the scatterers, and with the correlations between these two mechanisms: $\left\langle\Delta \varphi_{s}^{2}\right\rangle_{t, H, N}=\left\langle\Delta \varphi_{s, n}^{2}\right\rangle_{t, H, N}+\left\langle\Delta \varphi_{s, d}^{2}\right\rangle_{t, H, N}+\left\langle 2 \Delta \varphi_{s, n} \Delta \varphi_{s, d}\right\rangle_{t, H, N}$. 
Among the terms $\Delta \varphi_{s, d}^{2}, \Delta \varphi_{s, n}^{2}$, and $2 \Delta \varphi_{s, d} \Delta \varphi_{s, n}$, after averaging over time, only those which contain products $\tilde{f}_{0}\left(n /\left(v_{a} T\right)\right) \tilde{f}_{0}\left(m /\left(v_{a} T\right)\right)$ where $n+m=0$ survive. Further, for each frequency $n / T$, averaging over all free path lengths $l_{j}$ between consecutive scatterers and all scattering angles $\chi_{j}$ can be done in the same way as in Ref. 7 . Finally, averaging over the number of scattering events $N$ along the paths of length $s$ is straightforward. The result of averaging is

$$
\begin{aligned}
\left\langle\Delta \varphi_{s, n}^{2}\right\rangle_{t, H, N} & =\sum_{n=1}^{+\infty} \sin ^{2}\left(\frac{n \pi \tau}{T}\right) C_{n}(n), \\
\left\langle\Delta \varphi_{s, d}^{2}\right\rangle_{t, H, N} & =\sum_{n=1}^{+\infty} \sin ^{2}\left(\frac{n \pi \tau}{T}\right) C_{d}(n), \\
\left\langle 2 \Delta \varphi_{s, n} \Delta \varphi_{s, d}\right\rangle_{t, H, N} & =\sum_{n=1}^{+\infty} \sin ^{2}\left(\frac{n \pi \tau}{T}\right) C_{n, d}(n),
\end{aligned}
$$

where the C-terms $\left(C_{n}(n), C_{d}(n)\right.$, and $\left.C_{n, d}(n)\right)$ represent the amplitudes of the average of the squares of the phase terms at each ultrasound frequency:

$$
\begin{aligned}
C_{n}(n) & =\frac{\Lambda^{2}}{\pi^{2}}\left|\tilde{f}_{0}\left(\frac{n}{v_{a} T}\right)\right|^{2} \frac{\eta^{2}}{n^{2}}\left(k_{a} n l\right)^{2}\left[\left(\frac{s}{l}+1\right) \frac{G_{n}}{1-G_{n}}-\frac{G_{n}^{2}\left(1-G_{n} \exp \left[\left(G_{n}-1\right) s / l\right]\right)}{\left(1-G_{n}\right)^{2}}\right] \\
C_{d}(n) & =\frac{\Lambda^{2}}{\pi^{2}}\left|\tilde{f}_{0}\left(\frac{n}{v_{a} T}\right)\right|^{2} \frac{S_{n}^{2}}{n^{2}}\left[\frac{s}{3 l}-\frac{1-G_{n}^{-1} \exp \left[\left(G_{n}-1\right) s / l\right]-\exp [-s / l]\left(1-G_{n}^{-1}\right)}{\left(k_{a} n l\right)^{2}}\right] \\
C_{n, d}(n) & =\frac{\Lambda^{2}}{\pi^{2}}\left|\tilde{f}_{0}\left(\frac{n}{v_{a} T}\right)\right|^{2} \frac{2 \eta S_{n} \cos \left(\phi_{n}\right)}{n^{2}}\left[-\frac{s}{l}+\frac{G_{n}\left(1-\exp \left[\left(G_{n}-1\right) s / l\right]\right)}{1-G_{n}}\right] .
\end{aligned}
$$

In Eq. 11, $G_{n}$ is given by $\left(n k_{a} l\right)^{-1} \arctan \left(n k_{a} l\right)$. In a general case of the anisotropic scattering, expression similar to Eq. 10 could be found following the derivation from Ref. 7. Then, it can be shown that for a given path length $s$, the value of each C-term is approximately independent on particular values of the optical mean free path $l$ and anisotropy factor $g$, as long as the transport mean free path $l /(1-g)$ remains constant. This extends the conclusion about the similarity relation made in the case of large $k_{a} l$ values, ${ }^{7}$ to the case of small $k_{a} l$ values, too. For simplicity, in this work we will consider only isotropic scattering, noting that the anisotropic case can be approximately reduced to isotropic by replacing the $l$ in the isotropic equations with the value of $l_{t r}=l /(1-g)$. Also, we will frequently refer to the transport mean free path when making observations about the $k_{a} l$ dependence of C-terms, although the mean free path will be used in isotropic equations for simplicity.

\section{AUTOCORRELATION FUNCTION DEPENDENCE ON ULTRASOUND FREQUENCY}

The broadband ultrasound pulse has energy spread over a wide range of ultrasonic frequencies. In this section, we present some more detailed analysis of the ultrasound frequency dependence of the acousto-optical signal in optically diffusive media.

We focus here on the single frequency component in a general solution obtained in Sec. 2.2. For conciseness, we look at the special case of the train of ultrasonic pulses when it represents an actual monochromatic plane ultrasound wave $(\mathrm{CW})$. The $\mathrm{CW}$ case solution can be obtained from Eq. 10 if we first select the pulse shape function $f_{0}(u)$ to be equal to zero everywhere except in the interval $\left(-\pi / \hat{k}_{a}, \pi / \hat{k}_{a}\right)$, where it is equal to one sinusoidal cycle: $f_{0}(u)=\sin \left(\hat{k}_{a} u\right), \quad u \in\left(-\pi / \hat{k}_{a}, \pi / \hat{k}_{a}\right)$, and $f_{0}(u)=0$ elsewhere. Then, we take the limit $\hat{k}_{a} \rightarrow k_{a}$, where $k_{a}$ is the magnitude of the ultrasonic wave vector associated with the period between ultrasonic pulses. In the limiting case, the time dependence function $f(x, t)$ defined in Eq. 2 is reduced to a pure sinusoidal function. The Fourier transform of $f_{0}(u)$ for discreet frequencies $\nu=n /\left(v_{a} T\right)$, and in a limiting case $\hat{k}_{a} \rightarrow k_{a}$, 

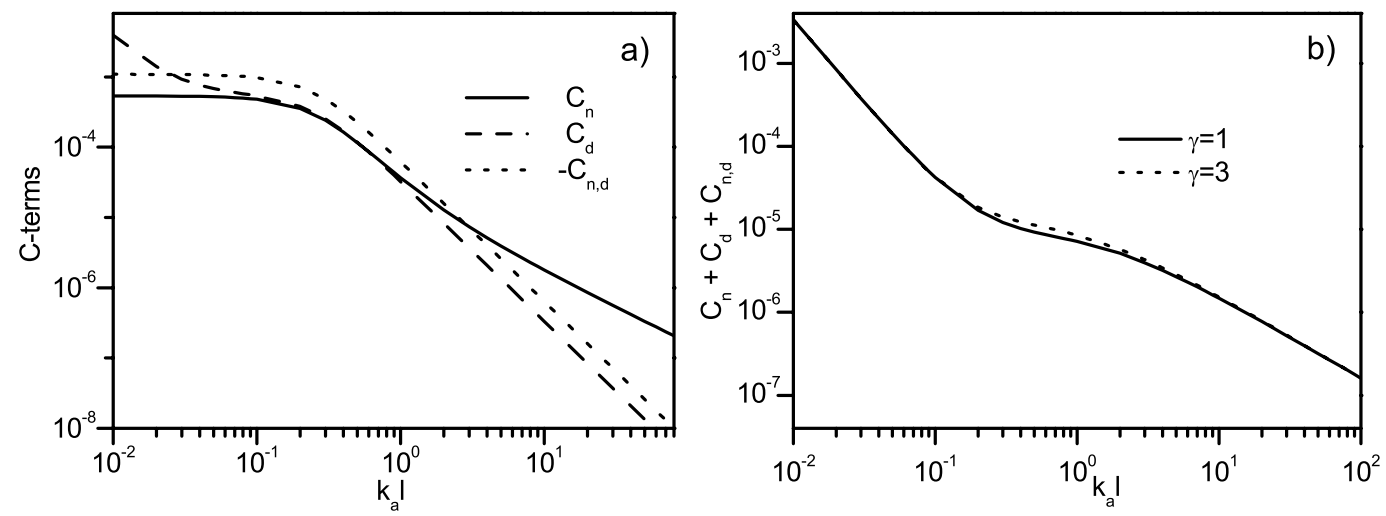

Figure 1. Dependence of the C-terms on the ultrasound frequency for $s / l=100$. a) Dependence of index of refraction term $C_{n}$, displacement of the scatterers term $C_{d}$, and mixed term $C_{n, d}$ multiplied by -1 for $\gamma=1$ value of the mass density ratio of scatterers. b) Sum of the C-terms, for two different values of the mass density ratio $\gamma$. Values of the parameters are: $l=1 \mathrm{~mm}, \Lambda=1 \mathrm{~m}^{-1}, \eta=0.32, S=1, \phi=0$, kinematic viscosity of water $\eta_{k}=10^{-6} \mathrm{~m}^{2} \mathrm{~s}^{-1}$, and scatterer radius $a_{0}=1 \mu \mathrm{m}$.

is zero for all $n$ except when $n=1$. For $n=1$, we have $\tilde{f}_{0}\left(1 /\left(v_{a} T\right)\right)=i v_{a} T / 2$, and set of equations 10 simplifies to the solution for the $\mathrm{CW}$ case

$$
\left\langle\Delta \varphi_{s, n}^{2}\right\rangle_{t, H, N}+\left\langle\Delta \varphi_{s, d}^{2}\right\rangle_{t, H, N}+\left\langle 2 \Delta \varphi_{s, n} \Delta \varphi_{s, d}\right\rangle_{t, H, N}=\sin ^{2}\left(\frac{1}{2} \omega_{a} \tau\right)\left[C_{n}+C_{d}+C_{n, d}\right]
$$

where

$$
\begin{aligned}
C_{n} & =\Lambda^{2} \frac{\eta^{2}}{k_{a}^{2}}\left(k_{a} l\right)^{2}\left[\left(\frac{s}{l}+1\right) \frac{G}{1-G}-\frac{G^{2}(1-G \exp [(G-1) s / l])}{(1-G)^{2}}\right] \\
C_{d} & =\Lambda^{2} \frac{S^{2}}{k_{a}^{2}}\left[\frac{s}{3 l}-\frac{G-\exp [(G-1) s / l]-\exp [-s / l](G-1)}{G\left(k_{a} l\right)^{2}}\right] \\
C_{n, d}(n) & =\Lambda^{2} \frac{2 \eta S \cos (\phi)}{k_{a}^{2}}\left[-\frac{s}{l}+\frac{G(1-\exp [(G-1) s / l])}{1-G}\right]
\end{aligned}
$$

In Eq. 13, the subscript $n$ is removed from $G_{n}, S_{n}$, and $\phi_{n}$, since all of them are calculated at the same ultrasound frequency, i.e. when $n=1$. These expressions are generalizations of the previously derived theory ${ }^{5,7}$ to cases where the optical transport mean free path is smaller than the ultrasonic wavelength. Therefore, in Eq. 13, not only the parts that are linear with the $s / l$ are presented, but, also, the terms which are a result of the strong correlation among the optical phase increments due to the different scattering events, and among the different optical free paths between consecutive scatterers. Another important difference is that we have significant correlation between the phase increments due to mechanism 1 and mechanism 2, unless the cosines of the phase lag between the ultrasound induced movement of the scatterers and the fluid is exactly zero. This correlation is represented in the mixed term [Eq. 13b], and it is not zero even for the large values of the $k_{a} l$ product when the correlations vanish between phase increments due to only mechanism 1 or only mechanism 2 .

Fig. 1(a) presents the ultrasound frequency dependence of C-terms in Eq. 13 along the optical path when $s / l=100$. The parameters used in calculation are: optical mean free path $l=1 \mathrm{~mm}$; elasto-optic coefficient of water at room temperature $\eta=0.32 ; \Lambda=1 \mathrm{~m}^{-1}$; and it is assumed that the scatterers are closely following the fluid displacement $(S=1, \phi=0)$. The term $C_{n, d}$ is multiplied by -1 to be presented on the same graph with the other two terms, although its value is negative and it actually cancels out, to some extent, the phase 
accumulations due to the individual contributions of the two mechanisms. It is important to notice that the ratio between each two C-terms in Eq. 13 for a given $s / l$ ratio depends only on the $k_{a} l$ product, up to the multiplication constant which depends on $\eta, S$, and $\cos (\phi)$.

The index of refraction term $C_{n}$, and displacement term $C_{d}$ have quite different behaviors at the opposite ends of the $k_{a} l$ range, as can be seen from Fig. 1(a). In general, except for some intermediate interval of the $k_{a} l$ values, $C_{d}$ is inversely proportional to the square of the ultrasound frequency, when the ultrasound pressure amplitude is constant. When the $k_{a} l$ product is small, scatterers along the optical path occupy a space volume where the ultrasound phase is nearly equal, unless the value of $s / l$ is very large. The $C_{d}$ term in that region behaves like it was caused by only one scatterer. On the other side of the $k_{a} l$ range, when the optical transport mean free path is greater than the ultrasound wavelength, phase increments between different scattering events are uncorrelated. In that region the $C_{d}$ term is equal to the sum of individual scattering contributions, which are all proportional to $k_{a}^{-2}$.

The correlations in the $C_{n}$ term were observed earlier ${ }^{5}$ in the intermediate range of the ultrasound frequencies. When $k_{a} l$ is low enough that all of the scatterers occupy the space with the similar ultrasound phase, then the increments from the different free paths add constructively. When $k_{a} l$ is sufficiently large, the optical phase increments due to mechanism 2 from the different free paths are completely uncorrelated, and $C_{n}$ is inversely proportional to the ultrasound frequency. Therefore, it eventually dominates all of the other contributions to modulation, when $k_{a} l$ is sufficiently large.

Finally, in a case of $C_{n, d}$, when $k_{a} l$ is sufficiently large, we also have an absence of correlation between the phase increments due to mechanisms 1 and 2 for the components of the optical path that do not share the same free path. However, the correlation between the phase increments due to mechanisms 1 and 2 for the same free path between two consecutive scatterers is always present.

Finally, we plot on Fig. 1(b) the ultrasonic frequency dependence of the sum $C$ of the optical phase accumulation terms $C_{n}, C_{d}$, and $C_{n, d}$, for two different relative mass densities of the optical scatterers $(\gamma=1$ and $\gamma=3$ ), and $s / l=100$. We choose the mean optical scattering free path to be $l=1 \mathrm{~mm}$, and the radius of the optical scatterers as $a_{0}=1 \mu \mathrm{m}$. Only in the range of intermediate $k_{a} l$ values, where both the phase and amplitude difference between the scatterers and fluid motion exist (for $\gamma=3$ ), and where the $C_{d}$ term contributes significantly to the value of $C$, does a discrepancy appear between the values of the $C$ term for different $\gamma$ values. We expect in most situations in real biological soft tissues that $\gamma$ is just slightly different from unity, in which case the observed discrepancy is not significant.

We mentioned earlier that when $k_{a} l$ is large, the $\mathrm{C}$ term is dominated by the value of the index of refraction term $C_{n}$, and it is dependent on the $k_{a}^{-1}$. In contrary, in a low $k_{a} l$ limit, the $C$ term behaves like the $C_{d}$ term at low values of $s / l$, i.e. like it is caused by the displacement contribution of only one scatterer.

\section{TRANSMISSION AND REFLECTION OF THE ACOUSTO-OPTICALLY MODULATED LIGHT INTENSITY IN A SLAB GEOMETRY}

\subsection{Slab equations}

In this section we present the analytical expression for the acousto-optical signal produced by the train of ultrasound pulses in the case of an infinitely wide optically scattering slab. Since it is possible to find a reasonably good analytical expression for the path length probability density function for both transmission and reflection slab geometry, a slab has been considered previously for various problems. ${ }^{3,5,6,18,23-25}$ We choose the $Z$ axis of the coordinate system to be perpendicular to the infinitely wide slab of thickness $d$. The indices of refraction of both the surrounding and scattering media are $n_{0}$. A plane ultrasonic wave propagates within the slab (in the $X-Y$ plane) and is assumed to fill the whole slab. We consider here only the transmission through the slab, where one side of the slab is irradiated by a plane electromagnetic wave, and a point detector measures the optical intensity on the side of the slab opposite to the light source. By solving the diffusion equation for this geometry, it is possible to find an expression ${ }^{5,23,25}$ for the photon path length probability density function $p(s)$ : 


$$
p_{T}(s)=K_{T}(s) \sum_{n=1}^{+\infty}\left\{\left[d(n)-z_{0}\right] \exp \left(-\frac{\left[d(n)-z_{0}\right]^{2}}{4 D s}\right)-\left[d(n)+z_{0}\right] \exp \left(-\frac{\left[d(n)+z_{0}\right]^{2}}{4 D s}\right)\right\}
$$

where $K_{T}(s)=\sinh \left(d_{0} \sqrt{\mu_{a} / D}\right) s^{-3 / 2} \exp \left[-\mu_{a} s\right](4 \pi D)^{-1 / 2} / \sinh \left(z_{0} \sqrt{\mu_{a} / D}\right)$. In Eq. 14, $d(n)=(2 n-1) d_{0}$, the diffusion constant is given by $D=\left[3\left(\mu_{a}+\mu_{s}\right)\right]^{-1} ; d_{0}$ is the distance between the two extrapolated boundaries of the slab; and $z_{0}$ is the location of the converted isotropic source from the extrapolated incident boundary of the slab. The distance between the extrapolated boundary and the corresponding real boundary of the slab is $l \gamma^{*}$, where $\gamma^{*}=0.7104$ and $l$ is the scattering mean free path $\left(l=1 / \mu_{s}\right)$. The converted isotropic source is one isotropic scattering mean free path into the slab. Therefore, $d_{0}=d+2 l \gamma^{*}$, and $z_{0}=l\left(1+\gamma^{*}\right)$.

The following expressions are needed in order to perform averaging of the terms in Eq. 11 over the pathlength probabilities:

$$
T_{s}=\int_{0}^{+\infty} p_{T}(s) s d s, \quad T_{e x p, n}=\int_{0}^{+\infty} p_{T}(s) \exp \left[-Q_{n} s\right] d s,
$$

where $Q_{n}=\left(1-G_{n}\right) / l$. The averaging of $\exp [-s / l]$, which appears in the $C_{d}$ term in Eq. $11 \mathrm{~b}$, can be done simply by changing the $Q_{n}$ value to $1 / l$, and the appropriate term will be named $T_{\exp 1}$. After calculating the integrals in Eq. 15 we have

$$
T_{s}=\frac{d_{0} \operatorname{coth}\left(d_{0} \sqrt{\mu_{a} / D}\right)-z_{0} \operatorname{coth}\left(z_{0} \sqrt{\mu_{a} / D}\right)}{2 \sqrt{\mu_{a} D}}, \quad T_{e x p, n}=\frac{\sinh \left(d_{0} \sqrt{\mu_{a} / D}\right)}{\sinh \left(z_{0} \sqrt{\mu_{a} / D}\right)} \times \frac{\sinh \left(z_{0} \sqrt{\left(\mu_{a}+Q_{n}\right) / D}\right)}{\sinh \left(d_{0} \sqrt{\left(\mu_{a}+Q_{n}\right) / D}\right)}
$$

If we denote with $\left\langle C_{n}(n)\right\rangle_{s, T},\left\langle C_{d}(n)\right\rangle_{s, T}$, and $\left\langle C_{n, d}(n)\right\rangle_{s, T}$ the averages of the appropriate C-terms in Eq. 11 over all of the path lengths in the transmission geometry, we have with the help of Eq. 16

$$
\begin{aligned}
\left\langle C_{n}(n)\right\rangle_{s, T} & =\frac{\Lambda^{2}}{\pi^{2}}\left|\tilde{f}_{0}\left(\frac{n}{v_{a} T}\right)\right|^{2} \frac{\eta^{2}}{n^{2}}\left(k_{a} n l\right)^{2}\left[\frac{G_{n}}{1-G_{n}}\left(\frac{T_{s}}{l}+1\right)-\frac{G_{n}^{2}\left(1-G_{n} T_{\exp , n}\right)}{\left(1-G_{n}\right)^{2}}\right], \\
\left\langle C_{d}(n)\right\rangle_{s, T} & =\frac{\Lambda^{2}}{\pi^{2}}\left|\tilde{f}_{0}\left(\frac{n}{v_{a} T}\right)\right|^{2} \frac{S_{n}^{2}}{n^{2}}\left[\frac{T_{s}}{3 l}-\frac{1-G_{n}^{-1} T_{\exp , n}-T_{\exp 1}\left(1-G_{n}^{-1}\right)}{\left(k_{a} n l\right)^{2}}\right], \\
\left\langle C_{n, d}(n)\right\rangle_{s, T} & =\frac{\Lambda^{2}}{\pi^{2}}\left|\tilde{f}_{0}\left(\frac{n}{v_{a} T}\right)\right|^{2} \frac{2 \eta S_{n} \cos \left(\phi_{n}\right)}{n^{2}}\left[-\frac{T_{s}}{l}+\frac{G_{n}\left(1-T_{\text {exp }, n}\right)}{1-G_{n}}\right] .
\end{aligned}
$$

\subsection{Various pulse shapes}

We present the effects of the acousto-optical modulation for two distinct types of the ultrasound pulse shapes. The Gaussian pulse shape (Pulse 1) is used as a representative of a pulse with the spectrum centered at the zero frequency. The second pulse shape function (Pulse 2) is produced by modulating the Pulse 1 profile with the cosines function, and it is a more realistic example of commonly generated ultrasound pulses. Fig. 2a presents the time profiles of the pulse shape functions, whose expressions are

$$
\begin{aligned}
f_{0, P 1}(u) & =\exp \left[-\frac{u^{2}}{2\left(\sigma v_{a} T\right)^{2}}\right] \\
f_{0, P 2}(u) & =\exp \left[-\frac{u^{2}}{2\left(\sigma v_{a} T\right)^{2}}\right] \cos \left(k_{u} u\right)
\end{aligned}
$$


In Eq. $18, v_{a}=1480 \mathrm{~ms}^{-1}$ is the ultrasound velocity in water; $T=20 \mu \mathrm{s}$ is the time period between pulses; and $\sigma=2.5 \cdot 10^{-3}$ is the constant which controls the relative width of each pulse compared to the distance between consecutive pulses, such that both pulses have similar bandwidth $\approx 5.3 \mathrm{MHz}$. In Pulse $2, k_{u}$ is the magnitude of the ultrasound wave vector associated with the $8 \mathrm{MHz}$ central frequency.

The black circles on Fig. 2c and 2d present the squares of the Fourier transforms of the ultrasound pulse profiles $f_{0, P 1}(u)$ and $f_{0, P 2}(u)$, for different ultrasound frequencies $n / T$, respectively. When calculating the acousto-optical effect, we use the optical wavelength $\lambda_{0}=0.5 \mu \mathrm{m}$, the optical index of refraction $n_{0}=1.33$, and the elasto-optic coefficient in water $\eta=0.32$. We also use the scattering mean free path $l=1 \mathrm{~mm}$, and the optical absorption coefficient $\mu_{a}=1 \mathrm{~cm}^{-1}$, which are in agreement with the typical optical transport mean free path and absorption coefficient in soft tissue. Since it is expected that in reality scatterers closely follow the ultrasound induced fluid motion, we use a relative scatterer density $\gamma$ equal to one. The scattering slab thickness is $d=4 \mathrm{~cm}$, and ultrasonic pressure amplitude $P_{0}=10^{5} \mathrm{~Pa}$. The parameters are also chosen such that the larger ultrasound wavelength in a spectrum is comparable with the slab thickness (or the source-detector distance), and the approximations involved in Eq. 4 are satisfied.

We define the scaling coefficient $C_{s c}(n)$, and the total scaling coefficient $\left\langle C_{s c}(n)\right\rangle_{s, T}$ in transmission geometry, at each ultrasound frequency $n / T$ as
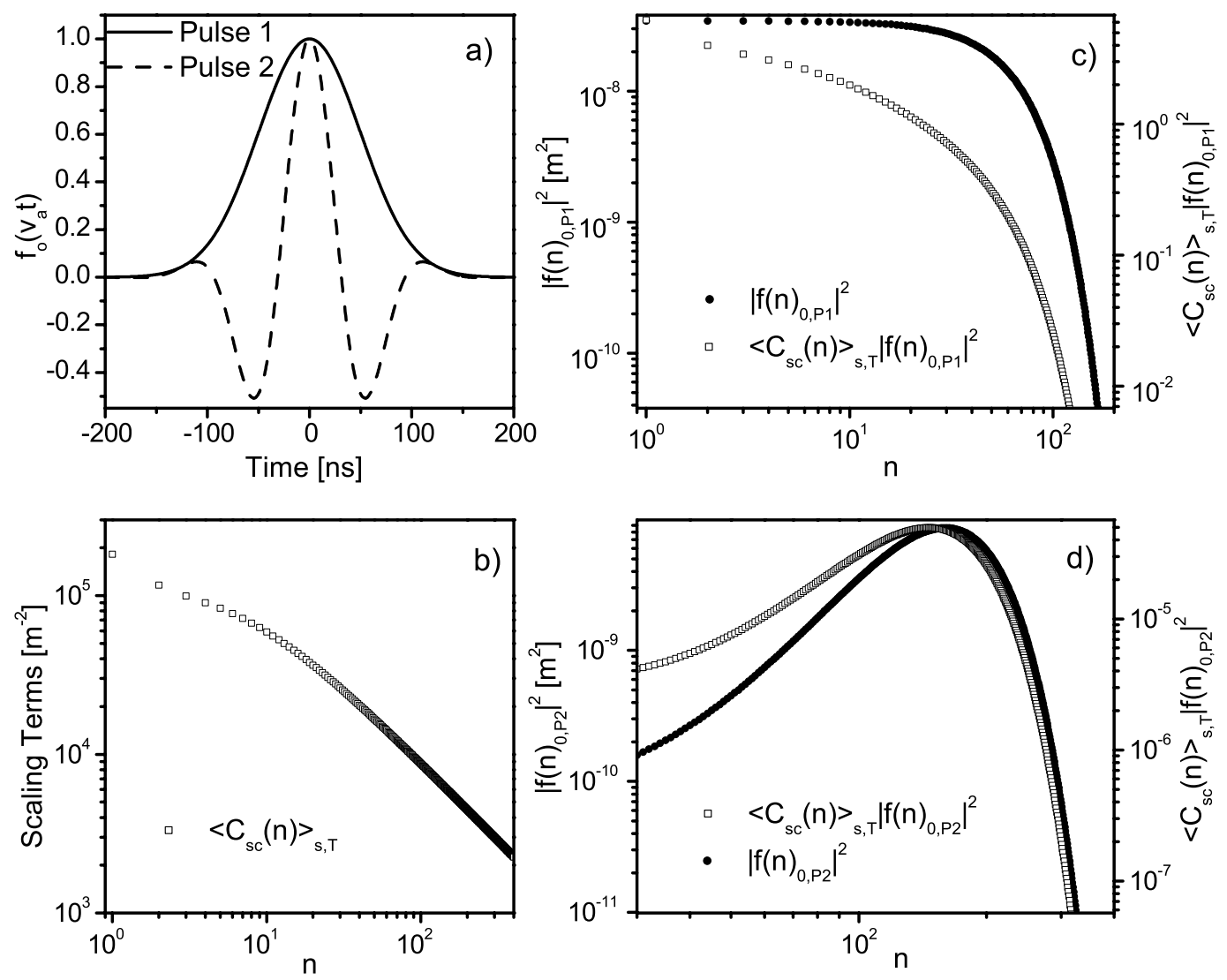

Figure 2. Frequency spectrums of pulses 1 and 2. (a) pulse time dependence; (b) ultrasound frequency dependence of the scaling terms; (c) power spectrum of the pulse 1 before and after multiplication with the scaling term; (d) power spectrum of the pulse 1 before and after multiplication with the scaling term; parameters used in calculation are: index of refraction in water $n_{0}=1.33$; optical wavelength $\lambda_{0}=0.5 \mu \mathrm{m}$; ultrasonic pressure amplitude $P_{0}=10^{5} \mathrm{~Pa}$; speed of sound in water $v_{a}=1480 \mathrm{~ms}^{-1}$; scattering mean free path $l=1 \mathrm{~mm}$; elasto-optic coefficient $\eta=0.32$; relative scatterer density $\gamma=1$; period between consecutive pulses $T=20 \mu \mathrm{s}$; pulse 2 central ultrasound frequency $T_{u}^{-1}=8 \mathrm{MHz} ; \sigma=2.5 \cdot 10^{-3}$. 


$$
\begin{aligned}
C_{s c}(n)\left|\tilde{f}\left(\frac{n}{v_{a} T}\right)\right|^{2} & =C_{n}(n)+C_{d}(n)+C_{n, d}(n), \\
\left\langle C_{s c}(n)\right\rangle_{s, T}\left|\tilde{f}\left(\frac{n}{v_{a} T}\right)\right|^{2} & =\left\langle C_{n}(n)\right\rangle_{s, T}+\left\langle C_{d}(n)\right\rangle_{s, T}+\left\langle C_{n, d}(n)\right\rangle_{s, T} .
\end{aligned}
$$

The open squares on Fig. $2 \mathrm{~b})$ present the frequency dependence of the total scaling coefficient $\left\langle C_{s c}(n)\right\rangle_{s, T}$, which is the result of the path length averaging of the scaling coefficient $C_{s c}(n)$. At each ultrasound frequency, the power spectral density of the acousto-optically modulated intensity is obtained by scaling the power spectral density of the train of pulses with this coefficient. The $\left\langle C_{s c}(n)\right\rangle_{s, T}$ behaves similarly to some $C_{s c}(n)$ term at the average value of the pathlength $s$. In a high frequency range, it is inversely proportional to the ultrasonic frequency, and in a low frequency range, depending on the average pathlength value, it might become inversely proportional to the ultrasound frequency square.

The open squares in Fig. 2c and Fig. 2d present the ultrasound frequency dependence of the power spectral density components given by $\left|\tilde{f}\left(n /\left(v_{a} T\right)\right)\right|^{2}\left\langle C_{s c}(n)\right\rangle_{s, T}$, in transmission geometry and for the Pulse 1 and Pulse 2 cases, respectively. Compared with the power spectra of the pulse shape functions (black circles), both pulses are attenuated more at the higher ultrasound frequencies due to the decay of the total scaling coefficient $\left\langle C_{s c}(n)\right\rangle_{s, T}$. Pulse 1 is attenuated strongly at higher frequencies, and it suffers a large reduction in bandwidth. The present theoretical model is not valid for very low values of the $k_{a} l$ product, and the concept of the infinite train of pulses allows us to avoid this part of the spectrum even in a case when the pulse shape function has very low frequency components, as in Pulse 1 case. Consequently, based on this model, it is difficult to predict the spectrum of the optical intensity after interaction with only one pulse with a similar shape. However, based on the presented theoretical derivations, it looks reasonable to us to expect a large bandwidth reduction for a pulse with a spectrum centered at zero frequency. In the case of Pulse 2, the frequency spectrum of the acousto-optically modulated light is slightly broadened for $\approx 0.3 \mathrm{MHz}$, and the central frequency is left-shifted by $0.7 \mathrm{MHz}$. Note also that the value of the total scaling coefficient $\left\langle C_{s c}(n)\right\rangle_{s, T}$ at the central frequency of Pulse 2 is several times smaller than its value at the lowest frequency in the spectrum.

\section{CONCLUSION}

In conclusion, we have presented an extension of the theory of acousto-optical modulation of the multiply scattered diffused light toward the small $k_{a} l$ values, where a strong correlation exists between the ultrasound induced optical phase increments associated with different components of the optical path. It is shown that an approximate similarity relation is valid for this extended range of $k_{a} l$ values. For large $k_{a} l$ values, an inverse linear dependence of the modulated signal on the ultrasound frequency is a consequence of the dominating effect of mechanism 2, while in the low $k_{a} l$ range, depending on the particular values of the average number of scattering events along the pathlength, the signal might be little dependent, or even inversely proportional to the square of the ultrasound frequency. The theory is also extended to account for complex scatterer movement in respect to surrounding fluid displacement. It is expected that in a cases involving the commonly used ultrasound pressures in medicine, the movement of the optical scatterers in soft biological tissues should not differ significantly from the movement of the surrounding tissue. In this situation, even for large values of the $k_{a} l$ product, a significant correlation between contributions of mechanism 1 and 2 exists. Finally, we derived an analytical solution for acousto-optical modulation when the train of the ultrasound pulses traverses the scattering media. Examples of two characteristic pulse shapes with zero and non-zero central frequencies are presented in the transmission and reflection geometries. It is shown that the ultrasound frequency dependence of the optical phase variations due to mechanisms 1 and 2 produces a nonuniform deviation of the pulse spectra, as well as decay of the modulated light power in the higher ultrasound frequency ranges.

\section{REFERENCES}

1. F. A. Marks, H. W. Tomlinson, and G. W. Brooksby, "A comprehensive approach to brest cancer detection using light: photon localization by ultrasound modulation and tissue characterization by spectral discrimination," in Proc. Soc. Photo-Opt. Instrum. Eng., 1888, pp. 500-510, 1993. 
2. L.-H. V. Wang, S. L. Jacques, and X. Zhao, "Continuous-wave ultrasonic modulation of scattered laser light to image objects in turbid media," Opt. Lett. 20, pp. 629-631, 1995.

3. W. Leutz and G. Maret, "Ultrasonic modulation of multiply scattered-light," Physica B 204, pp. 14-19, 1995.

4. M. Kempe, M. Larionov, D. Zaslavsky, and A. Z. Genack, "Acousto-optic tomography with multiply scattered light," J. Opt. Soc. Am. A 14, pp. 1151-1158, 1997.

5. L.-H. V. Wang, "Mechanisms of ultrasonic modulation of multiply scattered coherent light: an analytic model," Phys. Rev. Lett. 87, pp. 043903-(1-4), 2001.

6. L.-H. V. Wang, "Mechanisms of ultrasonic modulation of multiply scattered coherent light: a monte carlo model," Opt. Lett. 26, pp. 1191-1193, 2001.

7. S. Sakadzic and L.-H. V. Wang, "Ultrasonic modulation of multiply scattered coherent light: an analytical model for anisotropically scattering media," Phys. Rev. E 66, pp. 026603-(19), 2002.

8. A. Lev and B. Sfez, "In vivo demonstration of the ultrasound modulated light technique," J. Opt. Soc. Am. A 20, pp. 2347-2354, 2003.

9. S. Sakadzic and L.-H. V. Wang, "High-resolution ultrasound-modulated optical tomography in biological tissues," Opt. Lett 29, pp. 2770-2772, Dec 2004.

10. A. Lev and B. G. Sfez, "Pulsed ultrasound-modulated light tomography," Opt. Lett. 28, pp. 1549-1551, 2003.

11. T. W. Murray, L. Sui, G. Maguluri, R. A. Roy, A. Nieva, F. Blonigen, and C. A. DiMarzio, "Detection of ultrasound-modulated photons in diffuse media using the photorefractive effect," Opt. Lett 29, pp. 25092511, Nov 2004.

12. M. R. Maxey and J. J. Riley, "Equation of motion for a small rigid sphere in a nonuniform flow," Phys. Fluids 26, pp. 883-889, 1982.

13. E. E. Michaelidis, "Review - the transient equation of motion for particles, bubbles, and droplets," J. Fluid. Eng.-T. ASME 119, pp. 233-247, 1997.

14. A. T. Hjelmfelt and L. F. Mockros, "Motion of discrete particles in a turbulent fluid," Appl. Sci. Res. 16, pp. 149-161, 1966.

15. D. A. Siegel and A. J. Plueddemann, "The motion of a solid sphere in an oscillating flow: an evaluation of remotely sensed doppler velocity estimates in the sea," J. Atmos. Ocean. Tech. 8, pp. 296-304, 1991.

16. R. N. Zitter, "Ultrasonic diffraction of light by short acoustic pulses," J. Acoust. Soc. Amer. 43, pp. 864-870, 1968.

17. T. H. Neighbors and W. G. Mayer, "Asymmetric light-diffraction by pulsed ultrasonic-waves," J. Acoust. Soc. Amer. 74, pp. 146-152, 1983.

18. G. Maret and P. E. Wolf, "Multiple light-scattering from disordered media - the effect of brownian-motion of scatterers," Z. Phys. B 65, pp. 409-413, 1987.

19. D. J. Pine, D. A. Weitz, G. Maret, P. E. Wolf, E. Herbolzheimer, and P. M. Chakin, Scattering and localization of classical waves in random media, World Scientific Publishing Co. Pte. Ltd., Singapore, 1990.

20. M. Rosenbluh, M. Hoshen, I. Freund, and M. Kaveh, "Time evolution of universal optical fluctuations," Phys. Rev. Lett. 58, pp. 2754-2757, 1987.

21. B. J. Ackerson, R. L. Dougherty, N. M. Reguigui, and N. U, "Correlation transfer: application of radiative transfer solution methods to photon correlation problems," J. Thermophys. Heat Tr. 6, pp. 577-588, 1992.

22. J. W. Goodman, Statistical Optics, John Wiley \& Sons, New York, 1985.

23. M. S. Patterson, B. Chance, and B. C. Willson, "Time resolved reflectance and transmittance for the noninvasive measurement of tissue optical-properties," Appl. Opt. 28, pp. 2331-2336, 1989.

24. D. J. Pine, D. A. Weitz, P. M. Chaikin, and E. Herbolzheimer, "Diffusing-wave spectroscopy," Phys. Rev. Lett. 60, pp. 1134-1137, 1988.

25. A. Ishimaru, Wave Propagation and Scattering in Random Media, Academic, New York, 1978. 